\title{
Farmaconutrición de la vitamina B12 para COVID-19
}

\author{
Vitamin B12 Pharmaconutrition for COVID-19 \\ Farmaconutrição da vitamina B12 para COVID-19 \\ William Manzanares ${ }^{1 *}$, Gil Hardy²
}

Recibido: 28 de julio de 2020. Aceptado para publicación: 30 de agosto de 2020
Publicado en línea: 2 de septiembre de 2020

https://doi.org/10.35454/rncm.v4n1.187

\begin{abstract}
Resumen
La mortalidad por la enfermedad de COVID-19 es mayor en los ancianos, muchos de los cuales sucumben al síndrome de dificultad respiratoria aguda (SDRA) desencadenado por la infección viral. La vitamina B12 parenteral en dosis altas ofrece una nueva terapia prometedora para los pacientes con COVID-19 con SDRA por sepsis/choque séptico. Las propiedades antiinflamatorias y antioxidantes de la vitamina B12 y las transcobalaminas pueden modular la inflamación sistémica, que contribuye a la cascada de citocinas que conduce al SDRA. Se requieren estudios clínicos para establecer un régimen apropiado para administrar vitamina B12 como farmaconutriente, para pacientes críticamente enfermos con COVID-19.
\end{abstract}

Palabras clave: farmaconutrición, COVID-19, vitamina B12, SDRA, cuidados intensivos.

\begin{abstract}
Mortality from COVID-19 disease is much greater in the elderly, many of whom succumb to acute respiratory distress syndrome (ARDS) triggered by the viral infection. High dose parenteral vitamin B12 offers a promising novel therapy for those COVID-19 patients with ARDS from sepsis/ septic shock. The anti-inflammatory and antioxidant properties of vitamin B12 and transcobalamins can modulate the systemic inflammation contributing to the cytokine cascade that leads to ARDS. Clinical studies are now required to establish an appropriate regimen for administering vitamin B12 as pharmaconutrient for critically ill COVID-19 patients.
\end{abstract}

Keywords: Pharmaconutrition; COVID-19; Vitamin B12; ARDS; Critical Care.

\section{Resumo}

A mortalidade pela doença de Covid-19 é muito maior em idosos, muitos dos quais sucumbem à síndrome de dificuldade respiratória aguda (SDRA) desencadeada pela infecção viral. A vitamina B12 parenteral em altas doses oferece uma nova terapia promissora para pacientes com Covid-19 com SDRA decorrente de sepse / choque séptico. As propriedades antiinflamatórias e antioxidantes da vitamina B12 e das transcobalaminas podem modular a inflamação sistêmica que contribui para a cascata de citocinas que leva à SDRA.

Estudos clínicos são necessários para estabelecer um regime apropriado para administrar vitamina B12 como um farmaconutriente para pacientes criticamente doentes com Covid-19.

Palavras-chave:farmaconutrição, Covid-19, vitamina B12, SDRA, terapia intensiva.
Departamento de medicina crítica, Hospital de Clínicas Dr. Manuel Quintela, Escuela de Medicina, Universidad de la República de Uruguay (UDELAR). Montevideo, Uruguay.

*Correspondencia: Gil Hardy. gil.hardy50@gmail.com

\section{INTRODUCCIÓN}

La enfermedad del Coronavirus 2019 (COVID-19) es una pandemia mundial, que constituye uno de los mayores desafíos para la medicina de cuidados críticos. Según la Organización Mundial de la Salud (OMS),
2 Ipanema Research Trust. Auckland, Nueva Zelanda.

hasta el mes de junio de 2020 se produjeron 10 millones de casos y aproximadamente 500000 muertes en todo el mundo, con más de 40000 en el Reino Unido. La mortalidad ha sido muy elevada en los pacientes mayores de 70 años ( $25 \%$ en el Reino Unido), en particular en los que presentan comorbilidades previas a 
la enfermedad, como la diabetes mellitus, enfermedades cardiovasculares, obesidad, cáncer y enfermedades respiratorias crónicas ${ }^{(1)}$.

El síndrome de dificultad respiratoria aguda (SDRA) es un proceso relacionado con la sepsis y alimentado por una tormenta de citocinas, la cual se desencadena por una infección viral. El SDRA puede ser grave y poner en peligro la vida, ya que el paciente puede requerir ventilación mecánica y una estancia prolongada en la unidad de cuidados intensivos (UCI) y en el hospital. $\mathrm{Al}$ igual que en otras infecciones virales, los pacientes con COVID-19 presentan niveles muy altos de citocinas proinflamatorias, en particular de interleucinas 6 (IL-6), IL-1, IL-17 y el factor de necrosis tumoral alfa (TNF- $\alpha$ ). Los ancianos y otras personas con comorbilidades han mostrado una respuesta inflamatoria más agresiva, un fenómeno que hasta ahora no se ha comprendido bien. Lamentablemente, al no existir un tratamiento específico para la infección por COVID-19, la mortalidad general en la UCI sigue siendo inaceptablemente alta, superior al $60 \%$, lo que pone de relieve la urgente necesidad de nuevas estrategias terapéuticas.

La cobalamina o vitamina B12 es una de las ocho vitaminas hidrosolubles del grupo $\mathrm{B}$, que ayuda a mantener los glóbulos rojos oxigenados y previene la anemia megaloblástica. Una deficiencia puede causar cansancio, desorientación y puede estar asociada con enfermedades cardiovasculares y resistencia a la insulina. La vitamina B12 natural se encuentra casi exclusivamente en productos de origen animal como el pescado, la carne, los productos lácteos y los huevos, de los que se suelen obtener las necesidades diarias de 2,4 microgramos para los adultos $(2,6 \mathrm{mcg} / \mathrm{d}$ en el embarazo) y $1 \mathrm{mcg} / \mathrm{d}$ para los niños.

La B12 se absorbe en el íleon terminal donde se une a una proteína, llamada factor intrínseco (FI), que se produce en el estómago. Normalmente sólo se absorbe el 50 $\%$ de la B12 de la dieta, pero a medida que envejecemos nuestra capacidad de absorberla disminuye aún más. En consecuencia, los ancianos mal alimentados y aquellos a los que se les ha quitado el colon y/o parte del íleon terminal no absorberán suficiente B12 y necesitan complementar su dieta con entre 5 mcg y 20 mcg por día ${ }^{(2)}$.

La metilcobalamina, que representa el 75-90\% de la reserva corporal de vitamina B12 en circulación, es un portador reversible de radicales de metilo ${ }^{(3)}$. Tras su absorción en la circulación, las proteínas de transporte, denominadas transcobalaminas (TCS) aseguran que la B12 llegue a las enzimas clave en la mitocondria para el metabolismo de lípidos, carbohidratos y proteínas.
La transferencia del grupo metilo enzimático dependiente de la B12 es esencial para la síntesis de proteínas, donde la eliminación de un grupo metilo del folato de metilo forma homocisteína (HCYS) que se convierte en metionina para la síntesis de novo de un precursor del ADN. In vitro, la vitamina B12 tiene la capacidad de regular la producción de citocinas inflamatorias, inhibir la producción de peróxido intracelular, mantener los niveles de glutatión intracelular (GSH), y prevenir la apoptosis celular ${ }^{(4)}$.

\section{ESTADO DE LA VITAMINA B12 EN LOS ENFERMOS GRAVES}

La deficiencia y el agotamiento de la vitamina B12 son comunes en todo el mundo, en particular entre los vegetarianos y los ancianos, siendo más frecuentes en las poblaciones más pobres. Se cree que aproximadamente el $30 \%$ de las personas mayores de 60 años tienen una deficiencia, y más de 1,8 millones de ancianos estadounidenses se estiman en riesgo de deficiencia ${ }^{(1)}$. Actualmente no hay información sobre el estado de la vitamina B12 de los pacientes de COVID-19, y no hay recomendaciones específicas de suplementación para los pacientes críticos con SDRA debido al choque séptico resultante de COVID-19. Sin embargo, existen algunos datos sobre la relación entre la prevalencia de las deficiencias vitamínicas y la mortalidad hospitalaria en la UCI. Los bajos niveles de vitamina B12 son frecuentes en los pacientes que ingresan en la UCI, lo que afecta negativamente hasta el $15 \%$ de los pacientes mayores de 65 años. La deficiencia de B12 da lugar a trastornos hematológicos y neurológicos y puede ser un factor de riesgo independiente de la enfermedad de las arterias coronarias ${ }^{(5)}$.

Una asociación entre el estado de la vitamina B12 y la inflamación en los enfermos graves se demostró en el estudio realizado por Corcoran y colaboradores ${ }^{(6)}$, en el que los niveles de vitamina B12 diferían entre los pacientes que morían y los que sobrevivían. Además, dentro de las primeras 48 horas del ingreso en la UCI, se identificó una correlación positiva moderada entre la vitamina B12 y la proteína C-reactiva (PCR), un marcador de la respuesta de la fase aguda. Posteriormente, un segundo ensayo de cohorte del mismo grupo mostró una relación significativa entre la concentración de vitamina B12 y la puntuación de la Sequential OrganFailure Assessment (SOFA) $)^{(7)}$. Por lo tanto, el estado de la vitamina B12 parece reflejar la respuesta inflamatoria determinada por la PCR durante los primeros días de 
estancia en la UCI y ser un indicador de insuficiencia orgánica, evaluada por la puntuación SOFA, lo que podría ser un indicador de su potencial para aliviar el SDRA en pacientes COVID-19 gravemente enfermos.

La vitamina B12 disminuye significativamente después de la cirugía cardíaca ${ }^{(8)}$, lo que puede explicarse por su consumo con fines eritropoyéticos. El fenómeno constituye otro ejemplo de inflamación sistémica, caracterizada por el estrés oxidativo con la generación de radicales libres y el agotamiento de los antioxidantes en los enfermos graves, lo que sugiere que la suplementación podría mejorar el estado nutricional después de una intervención quirúrgica, en particular en los pacientes con una ingesta alimentaria subóptima. En un estudio de la UCI española ${ }^{(9)}$ se encontró una alta prevalencia de hiperhomocisteinemia, que es perjudicial para el endotelio y contribuye al estrés oxidativo. El HCYS fue mayor en los pacientes que recibieron menos de la ingesta recomendada de vitamina B12 y ácido fólico. En cambio, la suplementación adecuada de vitamina B12 y ácido fólico redujo el riesgo de hiperhomocisteinemia. Un apoyo adicional a la administración de suplementos proviene de los trabajos de Ploder y colaboradores ${ }^{(10)}$, quienes observaron un aumento de los niveles totales de HCYS en los pacientes de la UCI no supervivientes después de un traumatismo múltiple y una sepsis grave, mientras que los niveles de HCYS en los supervivientes se mantuvieron estables y bajos. Dado que la metionina se sintetiza a partir del HCYS por medio de 5MeTHF en una reacción irreversible, una deficiencia de B12 puede atrapar el folato en la forma de metilo. Así, concentraciones elevadas de folato pueden enmascarar los síntomas hematopéyicos de la deficiencia de B12 en los enfermos graves.

\section{VITAMINA B12: LA RESPUESTA INFLAMATORIA}

En una hipótesis innovadora, Wheatley ${ }^{(11,12)}$ ha descrito la vitamina como una "pimpinela escarlata" para la resolución potencial de la inflamación. En efecto, la vitamina B12 podría contribuir a la etiología del SDRA, en particular cuando se debe a una infección. El apoyo experimental para esta hipótesis proviene de Birch y colaboradores ${ }^{(3)}$, quienes demostraron elegantemente que las propiedades antioxidantes de la vitamina B12 implican una reacción con los radicales libres del oxígeno reactivo (ROS) y del nitrógeno (RNS), ejerciendo un efecto economizador de antioxidantes GSH e induciendo una respuesta al estrés. También hay pruebas de que la vitamina B12 regula a la baja los niveles de NF- $\kappa B$, que puede ser una importante molécula de señalización de la deficiencia de vitamina B12. Además, la metilcobalamina es capaz de suprimir la producción de IL-6. En el suero humano y en el líquido cefalorraquídeo, los bajos niveles de vitamina B12 se asociaron con un aumento concomitante del neurotóxico TNF- $a$ y una disminución del factor de crecimiento epidérmico (EGF). Además, Weinberg y colaboradores ${ }^{(13)}$ demostraron que la hidroxicobalamina (OH-B12), puede desempeñar un papel en la modulación de la función de la óxido nítrico sintasa y la síntesis de óxido nítrico (NO) in vivo. La anemia perniciosa se caracteriza por la deficiencia de vitamina $\mathrm{B} 12 \mathrm{y}$, por consiguiente, por los bajos niveles de GSH. En los pacientes con sepsis o choque séptico, los niveles de GSH disminuyen rápidamente, pero la suplementación de vitamina B12 tiene un efecto de ahorro de GSH, lo que aumenta la biodisponibilidad citosólica del antioxidante. Esto, a su vez, promueve la conversión a GSSG, y genera el seleniuro de hidrógeno ( $\mathrm{H} 2 \mathrm{Se}$ ) necesario para la síntesis de selenocisteína y selenoproteínas, como el glutatión peróxido $(\mathrm{GPx})^{(14)}$. La vitamina B12 también puede desempeñar un papel directo en la defensa inmunológica y la inflamación a través de una función bacteriostática de $\operatorname{TCS}^{(4)}$, que es capaz de modular la respuesta inflamatoria.

\section{LA VITAMINA B12, UN POTENCIAL FARMACÉUTICO PARA LOS SDRA}

Sobre la base de la hipótesis de Wheatley y de los datos en animales y clínicos, los posibles mecanismos mediante los cuales la vitamina B12 y la TCS podrían aliviar la inflamación sistémica grave que conduce al SDRA después de una sepsis grave o un choque séptico pueden resumirse de la siguiente manera: mediante la inhibición selectiva de la óxido nítrico sintasa (iNOS) y la reducción del exceso de NO; mediante la disminución de los radicales RNS y ROS; mediante el ahorro de GSH; mediante la estimulación de la fosforilación oxidativa, junto con el papel bacteriostático de la TCS durante la fagocitosis.

Dado su papel como antioxidante y antiinflamatorio, la B12 puede proteger contra la disfunción de múltiples órganos mediante la modulación de la actividad de ciertas citocinas, factores de crecimiento y otros sustratos. De ello se desprende que las inyecciones intravenosas o intramusculares de altas dosis podrían utilizarse en una estrategia novedosa contra el SDRA en los pacientes de 
COVID-19. Sin embargo, para considerar la vitamina B12 como un farmaconutriente, será necesario utilizar dosis más altas que las recomendadas actualmente para la terapia rutinaria de nutrición enteral (NE) o parenteral $(\mathrm{NP})^{(4)}$. Por consiguiente, todavía hay que abordar las cuestiones relativas a la dosis óptima, la hora de inicio, los días de tratamiento y el mejor método de administración de la vitamina B12: ya sea sola o en combinación con otros micronutrientes parenterales.

\section{VITAMINA B12: SEGURIDAD Y POSOLOGÍA}

Los productos multivitamínicos para la nutrición parenteral de los adultos contienen 5 o 6 ug de cianocobalamina, la forma sintética estable de la B12. Las formulaciones estándar de nutrición enteral (NE) contienen 3-6 $\mu \mathrm{g}$ por cada $1500 \mathrm{kcal} / \mathrm{d}$ de alimento, que es la dosis diaria recomendada de $\mathrm{NP} / \mathrm{NE}^{(15)}$. Aunque es aproximadamente el doble de la ingesta diaria recomendada (RDI) no hay evidencia de ninguna toxicidad. Además, durante la NP puede haber pérdidas significativas en la orina, ya que hasta el $25 \%$ de la B12 no ligada "pasa primero" por los riñones y la B12 secretada en la bilis puede no ser recapturada por los receptores ileales si el íleon ha sido resecado.

La vitamina B12 es relativamente no tóxica en dosis orales que exceden los requerimientos normales en 10000 veces. Con una alta tasa de excreción renal, se ha utilizado para tratar con éxito a los seres humanos con deficiencia de cobalamina y envenenamiento con cianuro con poca o ninguna evidencia de toxicidad. La dosis de vitamina B12 recomendada actualmente en el Formulario Nacional Británico (BNF) ${ }^{(16)}$ para el tratamiento de la anemia perniciosa y otras anemias macrocíticas sin disfunción neurológica es $1000 \mu \mathrm{g}$ de OH-B12 inicialmente administrada por vía intramuscular tres veces a la semana durante 2 semanas, $y$ luego una vez cada 3 meses. Sin embargo, en la UCI se utilizan infusiones antídotos más grandes de 5 a $15 \mathrm{~g}$ de $\mathrm{OH}-\mathrm{B} 12$ para tratar la intoxicación por cianuro sin efectos adversos significativos. Los conocimientos actuales nos permiten especular que la administración intravenosa de 5 gramos o más de OH-B12, alcanzaría niveles máximos en la sangre de más de 1000 micromolares con efectos secundarios mínimos. Los ensayos de farmacoterapia necesitarían monitorizar las concentraciones plasmáticas y séricas de la vitamina B12 total, el ácido metil-malónico y el HCYS como biomarcadores eficaces de los cambios en el suministro de vitamina B12 y en el metabolismo ${ }^{(17)}$.

\section{CONCLUSIÓN}

Los datos clínicos actuales de animales y humanos sugieren que una alta dosis de vitamina B12 parenteral puede ser un enfoque favorable para mejorar el estado antioxidante y antiinflamatorio de los pacientes de COVID-19 con SDRA derivado de la sepsis o el choque séptico. Las cobalaminas podrían ser agentes potencialmente útiles para inhibir la producción de NOS y NO, controlar la activación de NF-kB y su eventual supresión, y restaurar la óptima bacteriostasis y fagocitosis. En este escenario, la vitamina B12 y/o el TCS podrían modular la inflamación sistémica contribuyendo a la cascada de citocinas que conduce al SDRA. Podría administrarse con seguridad una terapia parenteral de vitamina B12 a corto plazo comparable a las altas dosis de hidroxicobalamina que se administran actualmente para la intoxicación por cianuro. Sin embargo, se requieren estudios clínicos para aclarar las cuestiones pendientes sobre la dosis y el momento de administración óptimos y mejor tolerados en pacientes COVID-19 gravemente enfermos con SDRA.

\section{Financiación}

Ninguna.

\section{Conflicto de intereses}

Los autores declaran no tener ningún tipo de conflicto de interés.

\section{Declaración de autoría}

GH y WM contribuyeron por igual al artículo. Los autores revisaron el artículo y validaron su versión final.

\section{Referencias bibliográficas}

1. World Health Organization. https://www.who.int/emergencies/diseases/novelcoronavirus-2019. [Accessed 28 June 2020]

2. Krautler B. Vitamin B12: chemistry and biochemistry. Biochem Soc Trans. 2005; 33(Pt 4): 806-10. doi: https://doi. org/10.1042/BST0330806.

3. Birch CS, Brasch NE, McCaddon A, Williams JHH. A novel role for vitamin $\mathrm{B}(12)$ : Cobalamins are intracellular antioxidants in vitro. Free Radic Biol Med. 2009; 47(2): 184-8. doi: https://doi.org/10.1016/j.freeradbiomed.2009.04.023.

4. Manzanares W, Hardy G. Vitamin B12: The Forgotten Micronutrient for Critical Care. Curr Opin Clin Nutr Metab Care. 2010;13(6):662-8. doi: https://doi.org/10.1097/ MCO.0b013e32833dfaec. 
5. Romain M, Sviri S, Linton DM, Stav I, van Heerden PV. The role of vitamin B12 in the critically ill-a review. Anaesth Intensive Care. 2016:44(4); 447-52. doi: https://doi.org/10 .1177/0310057X1604400410.

6. Corcoran TB, O'Neill MA, Webb SAR, Ho KM. Prevalence of vitamin deficiencies on admission: relationship to hospital mortality in critically ill patients. Anaesth Intensive Care. 2009; 37(2):254-60. doi: https://doi. org/10.1177/0310057X0903700215.

7. Corcoran TB, O’Neill MP, Webb SAR, Ho KM. Inflammation, vitamin deficiencies and organ failure in critically ill patients. Anaesth Intensive Care. 2009; 37(5): 740-7. doi: https://doi. org/10.1177/0310057X0903700510.

8. Racca V, Castiglioni P, Ripamonti V, Bertoli S, Calvo MG, Ferratini M. Nutrition markers in patients after heart surgery. JPEN J Parenter Enteral Nutr. 2010; 34(2): 143-50. doi: https://doi.org/10.1177/0148607109357627.

9. Abilés J, Pérez AR, Moratalla G, Castaño J, Rodríguez EM, Pérez de la CA et al. High prevalence of hyperhomocysteinemia in critically ill patients: Vascular damage and adequate vitamin intake. e-SPEN. 2008; 3: e240-e245. doi: https://doi. org/10.1016/j.eclnm.2008.06.011.

10. Ploder M, Kurz K, Spittler A, Neurauter G, Roth E, Fuchs D. Early increase of plasma homocysteine in sepsis patients with poor outcome._Mol Med. 2010: 16(11-12): 498-504. doi: https://doi.org/10.2119/molmed.2010.00008.

11. Wheatley C. A scarlet pimpernel for the resolution of inflammation? The role of supra-therapeutic doses of cobalamin, in the treatment of systemic inflammatory response syndrome (SIRS), sepsis, severe sepsis, and septic or traumatic shock. Med Hypotheses. 2006; 67:124-42. doi: https://doi. org/10.1016/j.mehy.2006.01.036.

12. Wheatley C. The return of the Scarlet Pimpernel: cobalamin in inflammation II-cobalamins can both selectively promote all three nitric oxide synthases (NOS), particularly iNOS and eNOS, and, as needed, selectively inhibit iNOS and nNOS. J Nutr Environ Med. 2007; 16(3-4):181-211. doi: https://doi. org/10.1080/10520290701791839.

13. Weinberg JB, Chen Y, Jiang N, Beasley BE, Salerno JC, Ghosh DK. Inhibition of nitric oxide synthase by cobalamins and cobinamides. Free Radic Biol Med. 2009; 46(12): 1626-32. doi: https://doi.org/10.1016/j.freeradbiomed.2009.03.017.

14. Manzanares W, Biestro A, Galusso F, Torre MH, Mañáy N, Facchin G, et al. High dose selenium for critically ill patients with systemic inflammation. Pharmacokinetics and pharmacodynamics of selenious acid: A pilot study. Nutrition. 2010; 26(6): 634-40. doi: https://doi.org/10.1016/j. nut.2009.06.022.

15. Vanek VW, Borum P, Buchman A, Fessler TA, Howard L, Jeejeebhoy $\mathrm{K}$ et al. ASPEN position paper: recommendations for changes in commercially available parenteral multivitamin and multi-trace element products. Nutr Clin Pract 2012; 27:440-491.

16. Joint Formulary Committee. British National Formulary (Ed.57) London: BMJ Group and Pharmaceutical Press 2009. http://www.medicinescomplete.com. [Accessed on 20.7.20].

17. Hoey L, Strain JJ, McNul H. Studies of biomarker responses to intervention with vitamin B-12: a systematic review of randomized controlled trials. Am J Clin Nutr. 2009; 89(6):1981S96S. doi: https://doi.org/10.3945/ajcn.2009.27230C. 